EPJ Web of Conferences 71, 00017 (2014)

DOI: $10.1051 /$ epjconf/20147100017

(C) Owned by the authors, published by EDP Sciences, 2014

\title{
International Masterclasses - bringing LHC data to school children
}

\author{
Uta Bilow $^{1 \mathrm{a}}$ and Michael Kobel ${ }^{1}$ on behalf of IPPOG ${ }^{2}$ \\ ${ }^{1}$ Institute of Nuclear and Particle Physics, TU Dresden, 01062 Dresden, Germany \\ ${ }^{2}$ IPPOG, CERN, Geneva, Switzerland, ippog.outreach@cern.ch
}

\begin{abstract}
International Masterclasses provide an opportunity for high school students to be "scientists for a day". 15- to 19-year-old students in countries around the whole world are invited to one of about 160 nearby universities or research centres for one day in order to take part in an authentic research process. Lectures from active scientists give insight in topics and methods of basic research at the fundaments of matter and forces, enabling the students to perform measurements on real data from CERN's Large Hadron Collider. At the end of each day, like in an international research collaboration, the participants join in a video conference with other students groups and CERN for discussion and combination of their results. In 2013, about 10.000 students from 37 countries participated in the program. International Masterclasses are organised by IPPOG (International Particle Physics Outreach Group) and have the potential to add valuable experiences to physics education in school environments.
\end{abstract}

\section{Bringing LHC Data to School Children}

With the start-up of the new and powerful particle accelerator LHC, a new era in research has dawned. The LHC is used by physicists to study the smallest known particles - the fundamental building blocks of all things. It will revolutionize our understanding of nature, from the minuscule world deep within atoms to the vastness of the Universe. Results of the experiments are followed with great interest, not only by physicists but also by the general public. International Masterclasses allow high school students to experience this research at close range. The basic idea of the annual program is to let students work as much as possible like real scientists.

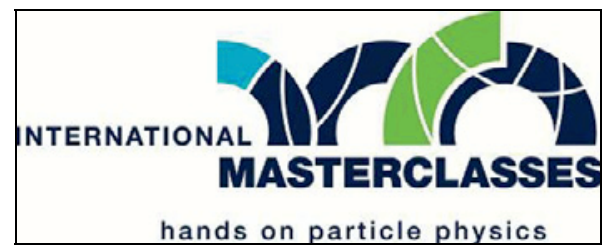

Figure 1. Logo International Masterclasses

\footnotetext{
${ }^{\text {a }}$ Corresponding author: uta.bilow@physik.tu-dresden.de
} 


\section{EPJ Web of Conferences}

International Masterclasses bridge the gap between science education at school and modern scientific research. High school students can explore the fundamental forces and building blocks of nature and are informed about the new age of exciting discoveries in particle physics. Moreover, they can actively take part in cutting-edge research and improve their understanding in science and the scientific research process. The program offers authentic experience and adds valuable experiences to physics education at school. In this way, the students' interest in science is stimulated, as an evaluation has shown.

\section{The Concept "Masterclass"}

In general a Masterclass is a class given to students of a particular discipline by an expert of that field. Usually Masterclasses are on arts - music, painting, drama - but here the subject is particle physics data analysis. The students work with an expert, a particle physicist, who gives them advice on how to analyse data from particle physics experiments. This unique hands-on approach ensures that the knowledge and insight that students acquire during the Masterclass is action-orientated.

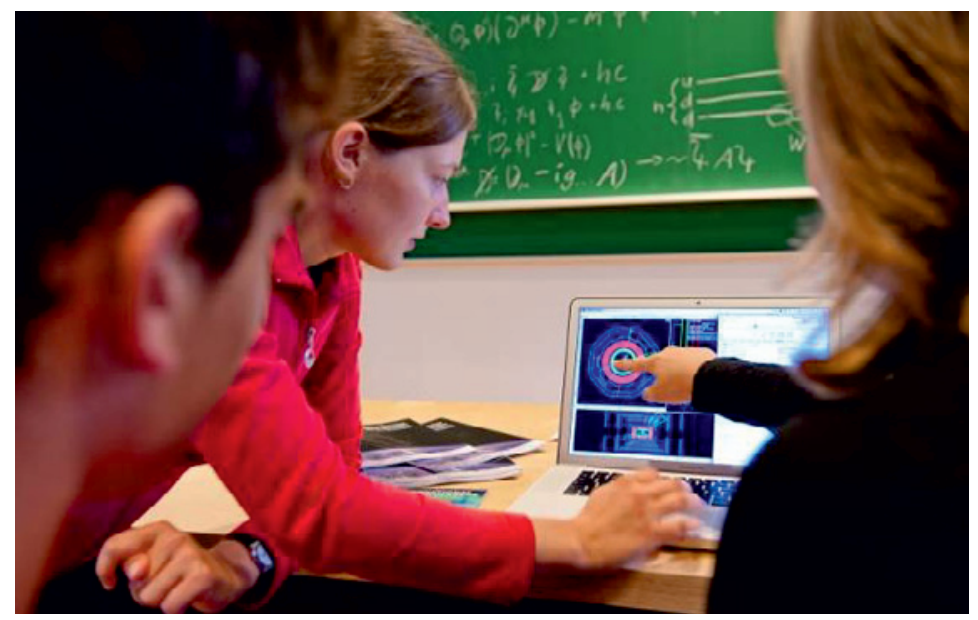

Figure 2. High school students in a particle physics Masterclass

\section{How the program developed}

Masterclasses in particle physics began in April 1997 in the United Kingdom as an initiative from Roger Barlow, Huddersfield University. The European program started in 2005, the World Year of Physics, when the European Particle Physics Outreach Group (EPPOG) adopted the idea and organised Masterclasses in 17 countries throughout Europe, with 3000 students participating. Since then the program is organised each year in spring and has grown steadily (Figure 3). In 2006, American students participated for the first time. Nowadays 37 countries from all continents (Figure 4) are represented in International Masterclasses, and the number of students participating is estimated at 10.000. The worldwide participation reflects the international collaboration in particle physics. 


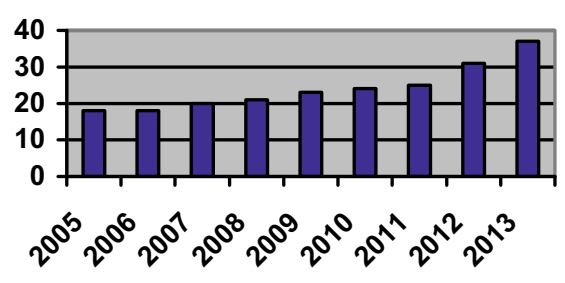

$\square$ Countries

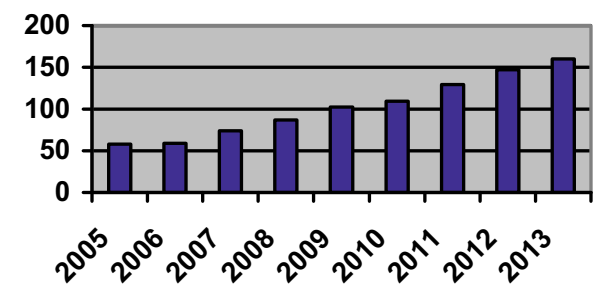

alnstitutes

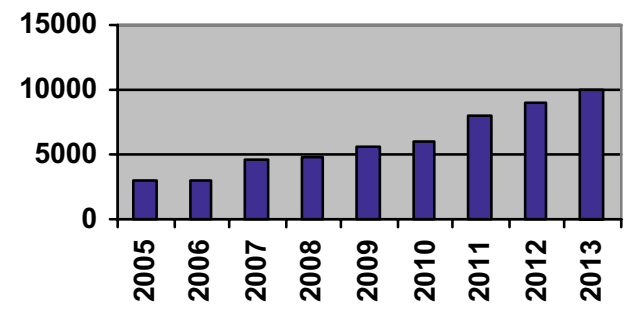

Students

Figure 3. From the beginning of the program in 2005 the numbers of participating countries, institutes, and students have constantly increased.
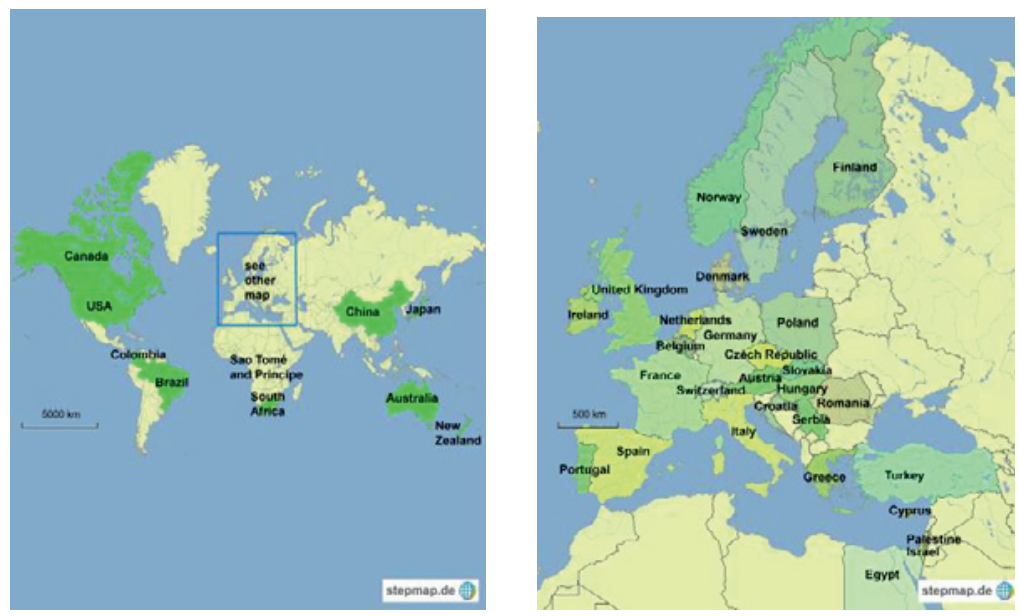

Figure 4. Countries participating in International Masterclasses. Left: worldwide, right: Europe

When International Masterclasses started, the new particle accelerator LHC at CERN (Geneva) was still under construction. Therefore students worked with data from former experiments at the Large Electron Positron (LEP) collider. The Large Hadron Collider (LHC) started colliding protons in 2009. Immediately, several groups developed new measurements for Masterclasses, where students could analyse the new data. In 2010, data from the LHC was included in Masterclasses for the first time. Since 2011 solely LHC data is analysed.

Various experiments at the LHC have made available data for Masterclasses, and several groups developed tools and measurement tasks. Students can work with data from ATLAS, CMS, or ALICE. 
In 2014, a measurement from LHCb will also be available. More experiments have expressed their interest to join the program in the next years.

\section{Masterclass Agenda}

Masterclasses are organized at more than 160 universities or research centers throughout the whole world. High school students aged 15 - 19 are invited to a research institute or a university to be "Scientist for one day". The size of the groups varies largely, from 15 up to 300, depending on the capacity of the inviting institution. The period for International Masterclasses covers four weeks in spring. Participating institutes choose their preferred date and sign in. Each day, up to 15 institutes can register.

\begin{tabular}{|c|c|c|c|c|}
\hline Tue 1.4. & Wed 2.4. & Thu 3.4. & Fri 4.4. & Sat 5.4. \\
\hline VC 1 & VC 1 & VC 1 & VC 1 & VC 1 \\
\hline ATLAS Z & ATLAS Z & ATLAS W & ATLAS W & ATLAS Z \\
\hline Athens I Jniv & Salentn & Strashnurg & Strashourg & Heraklinn \\
\hline I ercre & Tuehingen & Munich & Frlangen & I ishnn, IST-2 \\
\hline Grenoble & Barcelona & Roma Tre & Prešov & Braga \\
\hline Paris & London UC & Wuerzburg & Braga & Novi Sad \\
\hline Belgrad & Siegen & Wuppertal & & \\
\hline 5 & 5 & 5 & 1 & 1 \\
\hline Tue 1.4. & Wed 2.4 & Thu 3.4 . & Fri 4.4. & Sat 5.4 . \\
\hline VC 2 & VC 2 & VC 2 & VC 2 & VC 2 \\
\hline$\overline{A L I C E ~ R ~ A A ~}$ & CMS & ALICE & ATLA3 Z & ALICE \\
\hline Гrankfurt & Dudapest Wigner & São Paulo & Marseille & Warsaw, Univ. Techn \\
\hline Danmsladı GSI & Brussels Vije U. & Heidelbery & Udine & Geneva CERN \\
\hline \multirow[t]{3}{*}{ Piague CTU } & Filen<e & Oısay IPN & Durlmund & Cape Tuwiı \\
\hline & Catania & Nantes & Katowice & \\
\hline & & Geneva CERN & Grenoble & \\
\hline 3 & 4 & +2 & 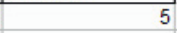 & 3 \\
\hline & & & & \\
\hline Iue 1.4 . & & I hu 3.4 . & & \\
\hline VC 3 & & VC 3 & & \\
\hline $\mathrm{LHCb}$ & & $\mathrm{LHCb}$ & & \\
\hline Marseille & & \begin{tabular}{|l} 
Dortmund \\
\end{tabular} & & \\
\hline Amsterdam & & Paris & & \\
\hline $\begin{array}{l}\text { Geneva CERN } \\
\end{array}$ & & Bucharest & & \\
\hline
\end{tabular}

Figure 5. Section of the schedule for International Masterclasses 2014. Each day, up to 15 institutes can sign up for one out of three video conferences (VC).

Although the participating institutions are able to configure some aspects of the event individually, each Masterclass follows a uniform scheme. In the morning of the day students listen to introductory talks about high energy physics. Presentations are adjusted to students' level and given by the masters - scientists familiar with outreach and education. In the lunch break, there is the opportunity for the students to talk to undergraduates, graduate students, and professors.

After the lunch break the practical part follows, which takes place on PCs. Students work with data from particle collisions recorded at the LHC on an own measurement. After an appropriate introduction to the programs they perform the measurement task autonomously, supported by the masters. At the end their findings are discussed with the group.

At the end of a Masterclass, students can expect a special highlight: an international video conference, where the students connect with more student groups from other countries and with a pair of moderators at CERN. All student groups of the given day have performed the same measurement, but with different data. Thus the video conference allows them to compare and combine their results and share their experiences. The focus is not just on results, but also on methods and research processes. The LHC is an example where the students can learn how a real research process proceeds.

\subsection{Lectures - conveying basic knowledge}

Many students visiting a Masterclass already have some ideas and knowledge about particle physics. Keywords such as CERN, quarks, black holes and the LHC are mentioned. This information is often 
based on media reports, as the field of particle physics is often only marginally represented in the curricula. Sound knowledge is usually not available. Therefore, in the morning lectures a basic introduction is provided. The talks cover the Standard Model of particle physics and the different types of elementary particles with their corresponding charges. Students hear about the four interactions, which mediate the dynamics of the known subatomic particles.

They usually are familiar with the best-known effect of these interactions, the electromagnetic force. This interaction, among other things, describes the attracting behaviour of oppositely charged particles, which causes the cohesion between the electron shell and positively charged atomic nucleus. New content, however, are often the strong force and the weak force. In addition, participants have to learn that force carrier act as mediators of interactions: For example, photons that are emitted by electrically charged particles in all directions and "captured" by other particles, are the force carriers of the electromagnetic force.

The lectures also have to cover, how particle accelerators and detectors operate and in which way different elementary particles interact with the detector. Students learn that particle accelerators are used on the one hand similar to optical microscopes, making possible the discovery of quarks in the interior of nucleons. On the other hand, the machines can be used to produce new particles in collisions of particles and their antiparticles: The annihilation of matter and antimatter creates force carriers, from which then in turn new, possibly even previously unknown particles can be formed.

\subsection{Measurements - hands-on activities}

Various measurements with LHC data have been developed for the use in Masterclasses. International Masterclasses 2014 will include six of them: ATLAS W path [1,2], ATLAS Z path [3,4], CMS W/Z path [5,6], Looking for Strange Particles in ALICE [7,8], ALICE Masterclass: The Nuclear Suppression Factor $\left(\mathrm{R}_{\mathrm{AA}}\right)$ [9], and LHCb Masterclass [10]. Participating institutes choose one of these measurements.

The basic idea of all measurements is a question related to particle physics. The outcome of each measurement should lead students to new and fundamental insights in this field. All tools and programs used in Masterclasses have been adapted to high school students' needs. For example, the measurements include event displays (Figure 6) where the detector signals are graphically implemented, or start at least with a visual analysis.

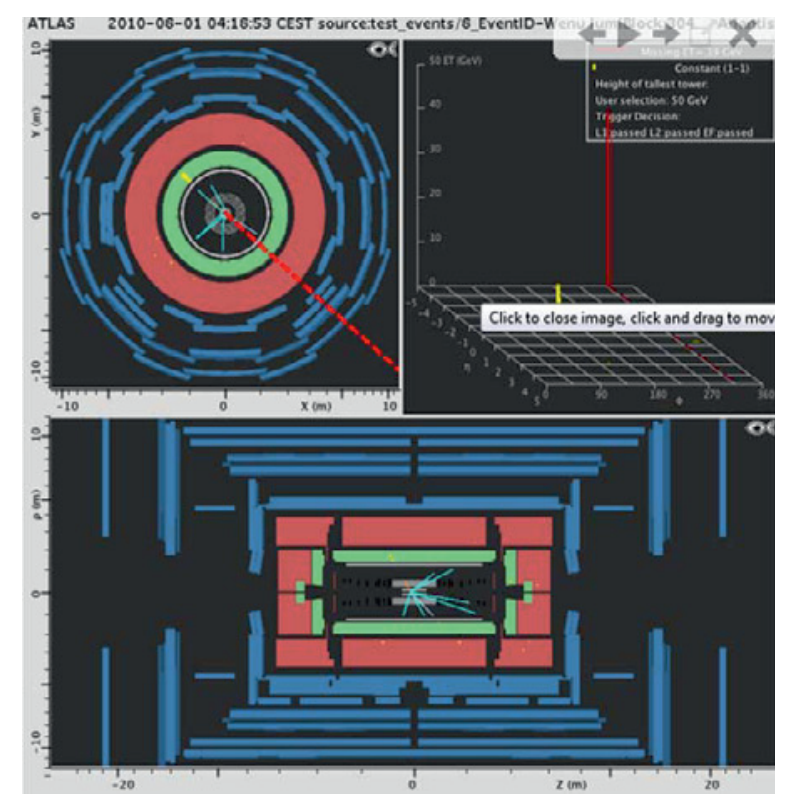

Figure 6. From the ATLAS W path measurement: Event display MINVERA, showing the decay of a W boson into a muon and a neutrino 
Students can follow the processing of results, and their results are presented in a graphical way (histograms or tables). For example, they learn to identify particles in event displays and to classify signal events. By simply counting the event types and comparing their relative abundances students achieve their result, thus copying the scientists' procedures. Other possibilities are measurements of the distribution of mass or angle or investigations on data quality.

One of the objectives of International Masterclasses is to let students follow up closely what the scientists are doing. Therefore consequently new measurement tasks are developed. In 2011, students could exploit known standard model processes, e.g. the $\mathrm{W}^{+} / \mathrm{W}^{-}$electric charge ratio (reflecting the dominance of positive u over negative d quarks inside the proton), or they were guided to understand the mass peaks of J/Psi and Z. In 2012, the discovery of new particles was added. Simulated data was included to give students the possibility to find extra $Z^{\prime}$ bosons or Higgs bosons decaying into WW. In 2013, real Higgs candidates have been included in the analysis.

\subsection{Video Conference - experiencing an international collaboration}

International Masterclasses are completed by a video conference. At the end of each day three to five student groups from different countries connect to CERN or Fermilab, where a pair of young scientists moderates the video conference (Figure 7). On the one hand this establishes a direct connection between the student groups and the research centre. On the other hand, the students receive an authentic impression of modern research. In particle physics, typically very large collaborations exist, including 3000 or even more scientists. For these scientists video conferences are a common working tool. A training for moderators is offered.

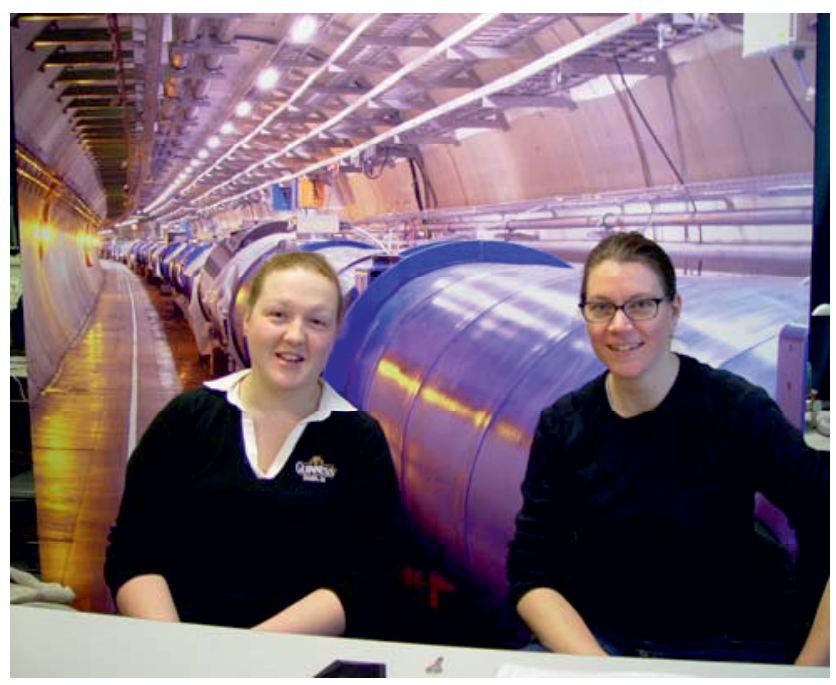

Figure 7. Moderators at CERN. A wallpaper in the teachers' lab helps to convey an authentic atmosphere.

Given a maximum of 15 institutes participating on one date, up to three video conferences are held in parallel. The video conferences have a duration of 60 minutes, are conducted in English and follow a fixed scheme. After a short welcome students present their results. All student groups in a given video conference have performed the same measurement, but with different data. Thus the video conference allows them to compare and combine their findings. After a discussion of the results, students have the opportunity to ask a broad range of questions to the moderators. As a final point, students complete a quiz with multiple choice questions about particle physics. The presentation is based on the famous TV-show "Who wants to be a millionaire?". Scoring is done by the students themselves, and the measure of the score is electron volts. 


\section{ICNFP 2013}

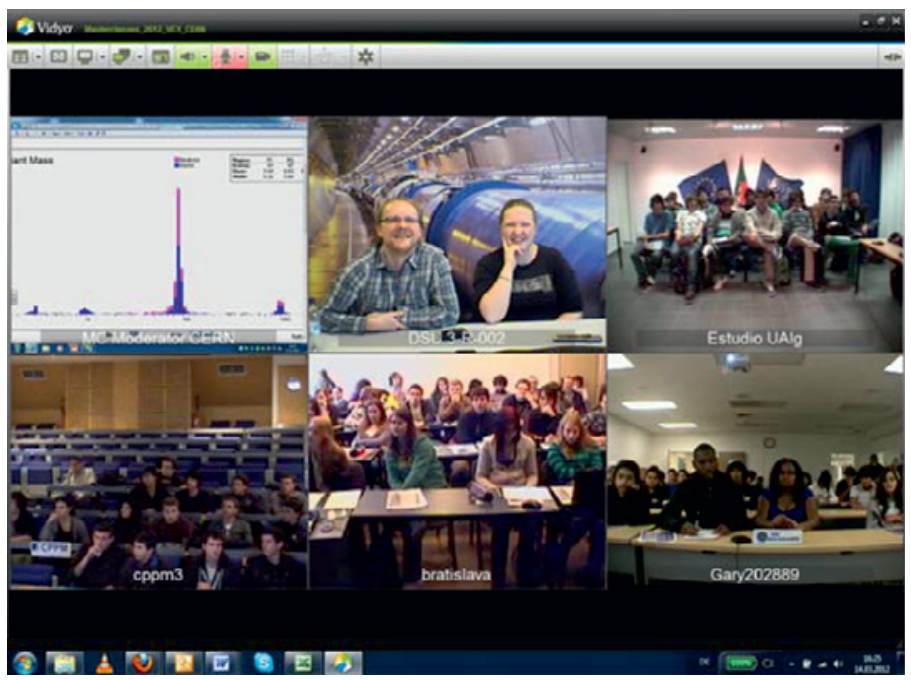

Figure 8. Screenshot from a video conference. Clockwise from top left: Combined mass histogram from ATLAS $\mathrm{Z}$ path measurement, moderators at CERN, students groups from Faro, Riverside (CA), Bratislava, and Marseille.

\section{Organisation}

International Masterclasses is a key project of IPPOG, a network of scientists, informal science educators and communication specialists working across the globe in informal science education and outreach for particle physics. The program is steered by a committee. The steering group includes project leader, coordinators, one person from each measurement, and other consultants. Further, a working group for the video conference has been established for the quality assurance of this item.

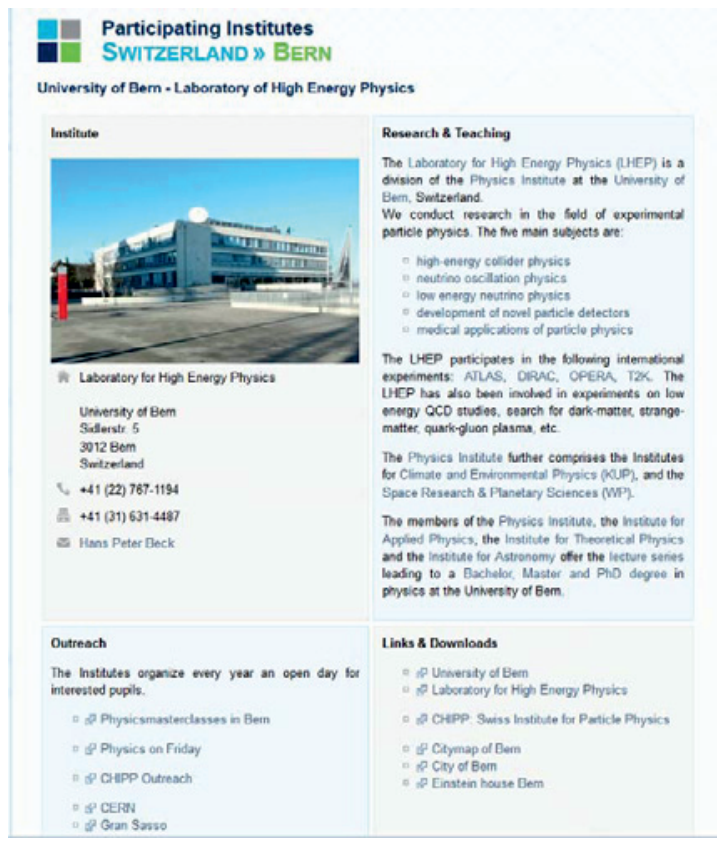

Figure 9. Profile from University of Bern on the website of International Masterclasses (www.physicsmasterclasses.org) 
International Masterclasses maintain a website (www.physicsmasterclasses.org) where all material is available. Measurements are free to use, not only in the framework of International Masterclasses., but for any educational purpose. All participating countries and institutes are presented with a profile, where contact details are included as well as information on research and teaching and outreach activities (Figure 9). Furthermore, details for the participating institutes are provided on the website, e.g. step-by-step lists for preparation, manuals, or corporate material to prepare invitation letters and certificates of participation. The conditions for participating institutes are manageable: Rooms (lecture hall, PC pool) and equipment for the video conference have to be provided. A scientist holds the lectures, tutors help during the measurement (1 master per 10 students). There are no financial requirements for the program.

\section{International Masterclasses - a successful program}

During a Masterclass students get to know the fundamental building blocks and interactions. They deal with current issues of basic research and methods of particle physics and learn about the importance of physics for the modern view of the world. The program aims to convey an authentic picture of the daily life of a scientist, to fascinate students about modern research in physics and to encourage their involvement with science. A refereed evaluation [11] investigated to what extent these objectives are achieved.
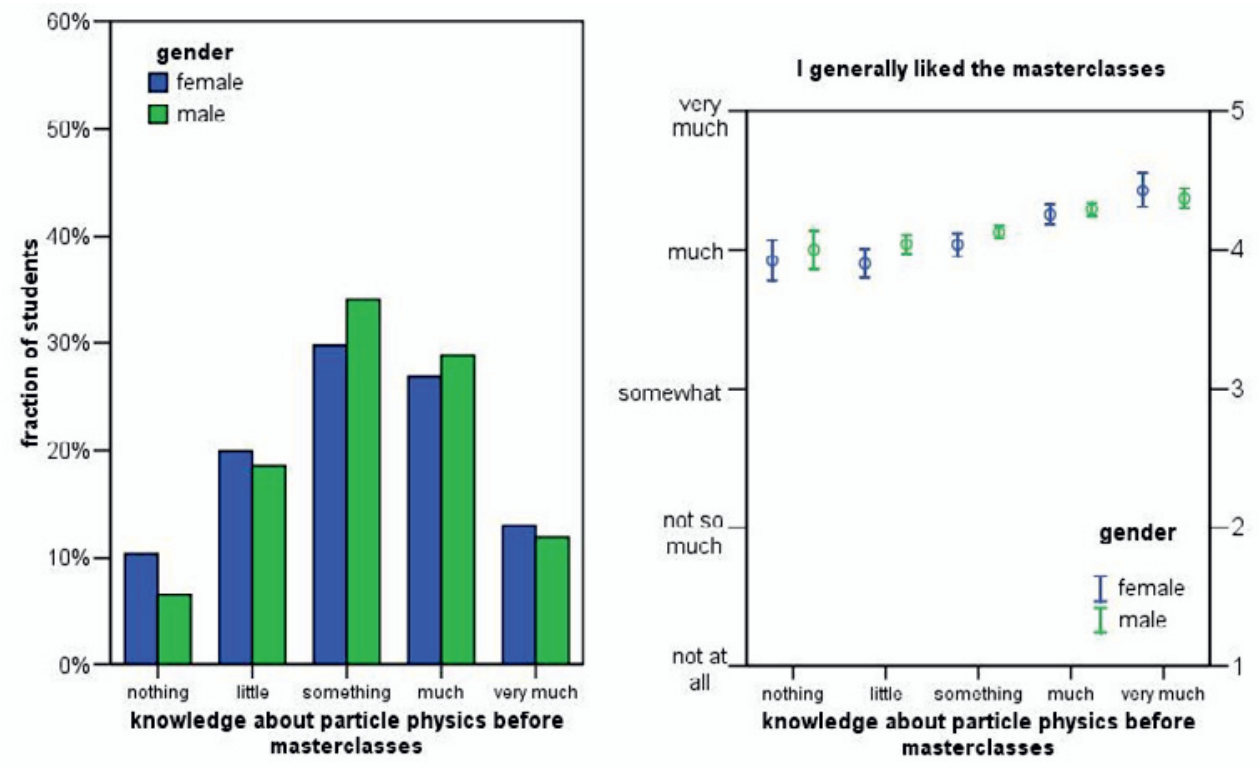

Figure 10. The students' pre-knowledge of particle physics and their appreciation of a Masterclass as a function of it.

1291 Students from 18 European countries were included in an evaluation study in 2005. The students' perception of the program was rated on a five-point Likert scale. An overwhelming majority of the students liked the Masterclass "much" or "very much" (82\%), while only $4 \%$ did not enjoy the program. Figure 10 shows that this high appreciation does not depend on the preknowledge of particle physics, and that there are no significant differences between the feedback from males and females. The perception of the lectures turned out to be the most important factor for the overall rating of a Masterclass. 

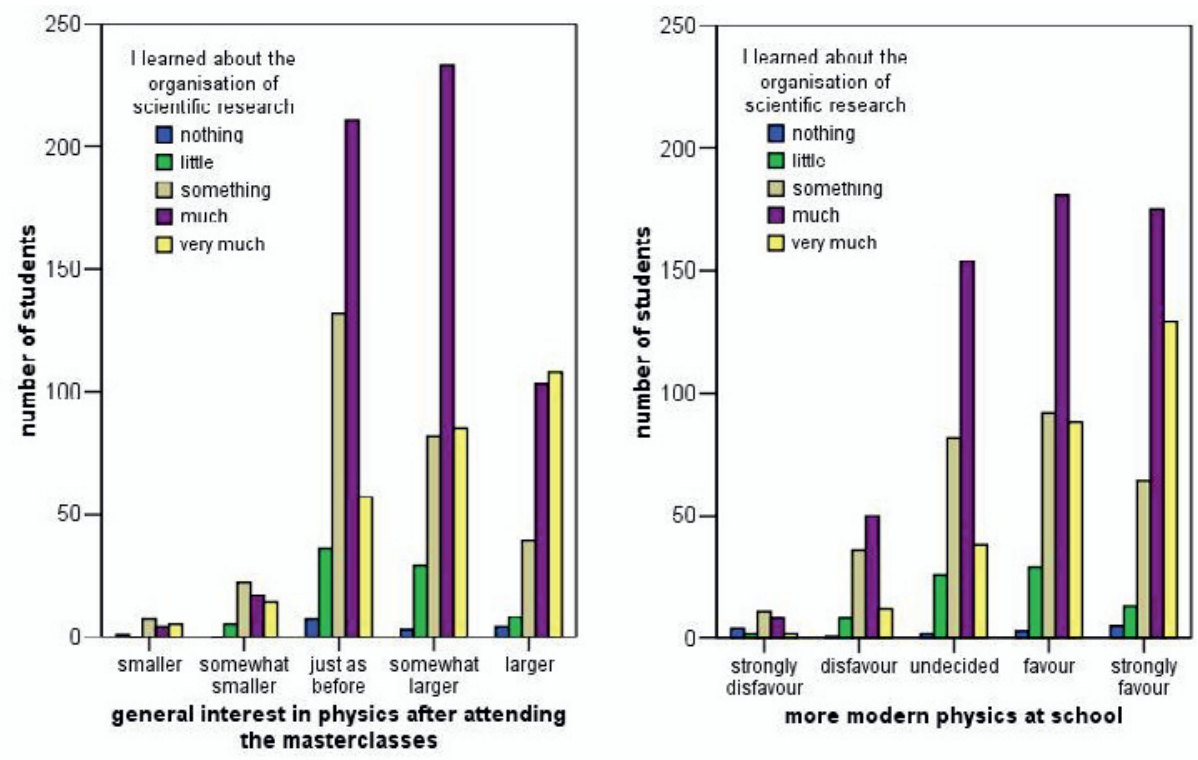

Figure 11. After attending a Masterclass the general interest in physics is increased and students wish to have modern physics at school.

Further questions asked about the increase in knowledge in particle physics and insight into to the way in which scientific research is organised. Students become the more interested in learning about physics and dealing with modern science in school the better they understand how scientific research is organized (Figure11). How much the students actually learned about particle physics only weakly influenced their overall appreciation of Masterclasses.

\section{Acknowledgements}

We are greatly indebted to CERN, QuarkNet, The German Helmholtz Alliance "Physics at the Terascale", TU Dresden, Fermilab, and the European Physical Society EPS - High Energy and Particle Physics Division for their financial and in-kind contributions. We are also very grateful to the members of IPPOG, to all groups developing measurements and tools, to the CERN Vidyo team for technical support of the video sessions, to all moderators at CERN, and to all the numerous scientists at universities and research institutes that organise Masterclasses at their institutes.

\section{References}

1. http://atlas.physicsmasterclasses.org/en/wpath.htm

2. U. Bilow, C. Hasterok, K. Jende, M. Kobel, C. Rudolph, J. Woithe, EPJ Web of Conferences 31 (2012) 01002

3. http://atlas.physicsmasterclasses.org/en/zpath.htm

4. M. K. Bugge, E. Gramstad, V. Morisbak, F. Ould-Saada, M. Pedersen, S. H. Raddum, EPJ Web of Conferences 31 (2012) 01002

5. http://cms.physicsmasterclasses.org/pages/cmswz.html

6. K. Cecire, EPJ Web of Conferences 31 (2012) 01002

7. http://alice.physicsmasterclasses.org/MasterClassWebpage.html

8. P. Foka, M. Janik, EPJ Web of Conferences 31 (2012) 01002

9. http://www-alice.gsi.de/masterclass/

10. http://lhcb-public.web.cern.ch/lhcb-public/en/LHCb-outreach/masterclasses/en/index.html

11. K.E. Johansson, M. Kobel, D. Hillebrandt, K. Engeln, M. Euler, Phys. Educ. 42, 636 (2007) 